\title{
John Millington Synge. L'île absolue
}

\section{Jérôme THELOT}

\section{OpenEdition}

\section{Journals}

Édition électronique

URL : http://journals.openedition.org/transtexts/228

DOI : 10.4000/transtexts.228

ISSN : 2105-2549

\section{Éditeur}

Gregory B. Lee

\section{Édition imprimée}

Date de publication : 1 janvier 2008

Pagination : 149-163

ISSN : 1771-2084

\section{Référence électronique}

Jérôme THELOT, « John Millington Synge. L'île absolue », Transtext(e)s Transcultures 跨文本跨文化 [En ligne], Hors série | 2008, mis en ligne le 14 septembre 2009, consulté le 04 mai 2019. URL : http:// journals.openedition.org/transtexts/228 ; DOI : 10.4000/transtexts.228 


\title{
John Millington Synge. L'île absolue
}

\author{
JEROME THELOT \\ Jean Moulin University - Lyon
}

W.B. Yeats described his friend, the dramatist John Millington Synge, in words stressing the relationship between Synge, the man of few words, and Synge, the man of a single place, Ireland, as "[t]hat rooted man, /Forgetting human words". Synge wrote a considerable amount of travel literature on various places in Ireland, having travelled on foot the length and breadth of the island. After some time spent on the European continent Europe, Synge returned to Ireland, and on the Aran Islands, through the translation from Gaelic into English of one of Ireland's oldest legends, the story of Deirdre, he created his own language in which he wrote all his plays. This invention, a discovery of himself and the universal meaning of his work would not have been possible without his encounter with the isles of Aran Islands.

J'ai décrit ailleurs comment nous découvrîmes que, lorsque nous franchissions la porte d'une chaumière paysanne, nous sortions de l'Europe au sens où l'on entend ce mot.

Yeats, Dramatis personae.

De John Synge, Yeats s'est souvenu dans l'un des grands poèmes de la fin de sa vie, en 1937, quand il eut retrouvé le visage de son ami, mort en 1909, dans le beau portrait qu'en avait peint son père, John Yeats, à la Galerie Municipale d'Art 
Moderne de Dublin, - et il écrit (c'est la dernière strophe du poème) : «Et voici là John Synge lui-même, / Cet homme enraciné 'à en perdre les mots'. » That rooted man, / 'Forgetting human words'.1 Yeats livre ici une clef pour entendre l'œuvre et le destin de Synge, et en plusieurs autres occasions il a souligné cette relation entre l'homme silencieux que Synge fut si souvent, à en perdre les mots, et l'homme d'un lieu qu'il fut tout autant, ce lieu en l'occurrence l'Irlande, et ses îles, ses vallées, ses grèves, et ses paysans enracinés.

Un premier signe de ce que cette relation entre parole et enracinement est en effet celle dans laquelle Synge a rencontré son drame et son énergie propres, c'est simplement d'abord qu'il a écrit - et cette part de son œuvre est considérable sur plusieurs lieux d'Irlande, des récits de marche d'un bourg à l'autre, d'une ferme à l'autre des comtés qui lui furent les plus chers, récits sur le Wicklow, sa région natale au sud de Dublin, sur le Kerry qui forme avec les îles Blaskets, comme il dit, « la pointe la plus occidentale de l'Europe», récits sur le Connemara et sur le Mayo, comtés de l'Ouest irlandais, les plus pauvres d'un pays pauvre, récits de ses rencontres avec les paysans dans leurs chaumières, de ses émotions devant la plus belle lumière du monde sur les falaises de l'Atlantique, témoignages du rapport passionnel, et aliéné, que les Irlandais entretiennent avec leur terre ingrate, colonisée, et avec leur paysage constamment surprenant parce qu'il passe, sans transition, du boccage le moins spectaculaire et le plus doux aux arêtes les plus impressionnantes, sublimes, des derniers escarpements du bout du monde. ${ }^{2}$ Le plus célèbre de ses ouvrages, c'est évidemment Les Îles Aran, que les lecteurs français connaissent par l'admirable traduction de Pierre Leyris. That rooted man, Synge fut requis par l'Irlande dès l'adolescence, où, selon son Autobiographie, «Tout ce qui est irlandais [lui] devint sacré », et il ajoute : "comme si j'étais tombé amoureux d'une déesse » (fallen in love with a goddess). ${ }^{3}$ Yeats assure dans ses essais que ce lien à l'Irlande, et plus précisément à « ce qu'il y avait de sauvage dans le peuple et dans 'les vallées aux flancs gris de l'hiver' », n'était pas seulement un fait psychologique et existentiel, mais le cœur de la poétique de son ami et de ses possibilités d'écrivain : ${ }^{4}$ «e doute, dit-il, qu'il eût jamais écrit s'il n'avait écrit de l'Irlande et pour elle, et je sais qu'il pensait qu'il n'est d'art créateur qui ne trouve son origine en une telle préoccupation. ${ }^{5}$ Dans la Préface que Synge a donnée à sa pièce la plus fameuse, Le Baladin du monde occidental, il écrit pareillement : «En

\footnotetext{
${ }^{1}$ «The Municipal Gallery Revisited», Quarante-cinq poèmes de Yeats, suivis de La Résurrection, Traduction, introduction et notes d'Yves Bonnefoy, Hermann, 1989, p. 158-159.

${ }^{2}$ John M. Synge, «Dans l'Ouest du Kerry », Dans le Wicklow, L'Ouest du Kerry et le Connemara, Traduit de l'anglais par Bernard Hœpffner, Castelnau-le-Lez, Climats, 1996, p. 78.

${ }^{3}$ Cité par Robin Skelton, J. M. Synge and his World, Thames and Hudson, 1971, p. 20.

${ }^{4}$ W. B. Yeats, « J. M. Synge et l'Irlande de son temps », texte reproduit dans J. M. Synge, Le Baladin du Monde Occidental, Traduction de Françoise Morvan, Éditions Folle Avoine, 1993, p. 27.

${ }^{5}$ Yeats., p. 26.
} 
Irlande, pour quelques années encore, nous avons une imagination populaire ardente, superbe et tendre; ainsi, ceux d'entre nous qui désirent écrire ont-ils d'entrée de jeu une chance qui fait défaut aux écrivains des régions où la source de la vie locale a été oubliée, où la moisson n'est plus qu'un souvenir, où la paille a été changée en brique. ${ }^{6} \mathrm{Il}$ n'est pas une des pièces de Synge qui, comme ses récits de marcheur, n'ait pour principal acteur le lieu du drame, le lieu en tant qu'il instaure le verbe enraciné des personnages. Le titre en français, Le Baladin du monde occidental, trahit cette détermination de la parole par l'enracinement local. The Playboy of the western world, le titre en anglais, suggère le vrai sens de la pièce pourvu qu'on entende correctement les mots playboy et western world, comme le réclame avec raison Françoise Morvan, qui a merveilleusement traduit le théâtre entier de l'écrivain. "Le mot "playboy" [...] appartient à l'argot anglo-irlandais. Son sens exact [...] est "farceur, raconteur de blague, mystificateur [...] personne qui truque, qui simule." [...] a) celui avec qui l'on plaisante; b) celui qui joue [...]; c) celui qui a le goût du jeu. » ${ }^{7}$ De sorte que ce playboy, qu'on ne saurait mieux rendre que ne l'a fait Françoise Morvan par "Le beau parleur», est une allégorie de la puissance poétique, de l'invention verbale, et des enchantements et des enchaînements liés à celle-ci. Le western world, d'autre part, «ne désigne pas le monde occidental mais l'ouest de l'Irlande, la partie gaélique, pauvre, tournée vers l'Amérique, opposée à l'est, riche, tourné vers l'Europe. $»^{8}$ The playboy of the Western World, donc, cela veut dire : Le Beau parleur des terres de l'ouest, à savoir des terres du Mayo et du Connemara où Christy Mahon, baratineur provisoirement génial qui allégorise la poésie aussi ravissante que désolée, arrive en vagabond muni seulement de sa parole et de son origine lointaine, puisqu'il vient, lui, des terres du sud, celles du Kerry, où, selon Yeats, la langue est la plus savoureuse, comme doit l'être selon Synge celle du théâtre, "aussi pleine de suc qu'une pomme ou qu'une noix.» ${ }^{9}$ Or voici donc la scène inaugurale de tout ce théâtre, la scène vraiment emblématique du rapport entre l'homme rooted et l'homme de paroles - cette scène que dans son expérience de marcheur et de témoin Synge a lui-même fréquentée dans les petites fermes de l'Irlande -, où quelqu'un, un vagabond d'abord silencieux, entre dans une maison pauvre de nulle part, au bord de l'océan qui gronde ou dans une vallée la plus reculée sous le ciel qui change. De cette scène, la puissance de suggestion est formidable. Car si l'œuvre dramatique de Synge doit son unité à sa langue, cette langue elle-même doit ses traits à cette maison du western world, chaumière ou pub, où n'importe qui n'importe quand, comme le playboy, peut, devant ses hôtes, soudain devenir ce qu'il est, un enchanteur et un faussaire, un beau parleur qui pour

\footnotetext{
${ }^{6}$ Yeats, p. 56

${ }^{7}$ Françoise Morvan, p. 11.

${ }^{8}$ Françoise Morvan, p. 11.

${ }^{9}$ Françoise Morvan, p. 56.
} 
le temps de son séjour, et rien qu'en parlant, ravive les apparences et renflamme l'amour dans cette maison alors plus vaste que le monde.

\section{La maison sublime}

De ces maisons comme il ne s'en trouve plus guère aujourd'hui en Irlande, les forces de police, du milieu du XIXe jusqu'au début du XXe siècle, expulsaient brutalement les habitants quand les fermages n'étaient pas payés, exécutant les volontés de propriétaires terriens autorisés par les lois iniques de la politique des colons. Maison poignante - symbole de la poésie selon Synge - et semblable à celle par la vertu de laquelle se produisent les drames de ses deux premières pièces : Cavaliers de la mer (Riders to the sea), et L'ombre de la vallée (In the Shadow of the glen), écrites l'une et l'autre en 1902. Voici les indications scéniques données pour le décor de ces drames : pour Riders to the sea : "L'action se passe dans une île à l'ouest de l'Irlande. / La cuisine d'une chaumière." Pour In the shadow of the glen : "La dernière chaumière au bout d'une longue vallée dans le comté de Wicklow. / La cuisine de la chaumière; à droite un feu de tourbe. » Ce décor se retrouvera dans les deux dernières pièces, Le Baladin et Deirdre des douleurs : une maison fermée autour d'un feu de tourbe, une chaumière pauvre, dont la pauvreté est pourtant aussi éloignée - comme on lit dans Les Îles Aran - de la «nullité des riches » que de la "sordidité des pauvres", maison donc qui rend possible un rapport au monde non pas d'abord politique mais d'emblée ontologique. ${ }^{10}$ La misère y est grande, évidemment, et même insupportable; un paysan du Connemara s'en ouvre à Synge : "Je suis depuis presque vingt ans sur ce bout de rocher qu'un chien ne voudrait même pas regarder, où les cochons meurent, où les pommes de terre meurent, même les juges et les gens de qualité viennent ici et baissent les fermages après avoir vu comment l'Atlantique sauvage se précipite contre ces satanés rochers. [...] Et je ne sais pas comment je vais continuer à vivre dans cet endroit que le Seigneur a créé en dernier, je crois bien, après tout le reste.» ${ }^{11}$ Mais cette misère n'empêche pas une autre sorte de richesse, comme si le malheur existentiel n'était pas incompatible avec une adhésion ontologique dont le poète témoigne : "Les fermiers, dit Synge, avec leur humour et leur simplicité, avec les fermes grises où ils vivent, jouissent, au sens réel, 'de richesses infinies dans une petite pièce' ».12 Dans cette petite pièce - une cuisine avec sa cheminée où le drame aura lieu, et donnant parfois, à côté, sur une inner room où les hommes dorment avec les bêtes - on se

\footnotetext{
${ }^{10}$ Les Îles Aran, Traduit par Piere Leyris, Castelnau-le-Lez, Éditions Climats, 1990, p. 78.

${ }^{11}$ Dans le Wicklow, L'Ouest du Kerry et le Connemara, p. 173-174.

${ }^{12}$ Dans le Wicklow, L'Ouest du Kerry et le Connemara, p. 70.
} 
retrouve le soir pour redire les malheurs du pays, réciter des poèmes extravagants, répéter des histoires de fées, de fous, d'émigrations et de naufrages. Ou bien, à toute heure, on y accueille le vagabond sans abri et même le rétameur.

La maison est ainsi la condition de possibilité de l'échange entre les hommes, par lequel sa précarité trouve sa sanctification : lieu du partage de la désolation commune, de l'égalité dans la misère, lieu de l'exposition de chacun au fait nu d'exister, à l'existence dure solidairement vécue. Aux Îles Aran, il est arrivé que Synge fût enfermé seul dans la maison, - et il raconte : «En me levant ce matin, je découvris que les gens étaient partis à la messe en bouclant du dehors la porte de la cuisine, si bien que je ne pouvais pas l'ouvrir pour me donner de la lumière. Je suis tellement habitué à me tenir là avec les autres que je n'ai jamais ressenti encore la chambre comme un endroit où n'importe qui pourrait vivre et travailler seul. Après un temps d'attente avec juste assez de lumière venue de la cheminée pour me laisser voir les chevrons et la grisaille des murs, je fus pris d'une tristesse inexprimable, car je sentais que ce petit coin de la surface du monde, et les gens qui y vivaient, jouissaient d'une paix et d'une dignité dont nous sommes retranchés pour toujours.» ${ }^{13}$ Ce petit coin de la surface du monde est ainsi, proprement, sublime. J'emploie le mot sublime ici au sens de Burke - Burke qui était irlandais, que Synge a lu (il le mentionne dans l'un de ses articles sur l'histoire de la littérature irlandaise), et dont le concept de «delight», nommant l'émotion du sublime, peut servir à décrire ce plaisir compliquée d'effroi, ce bonheur ontologique accru par le malheur existentiel, que Synge a trouvés dans la maison du western world. Le sublime - ou la terreur délicieuse, ou le soulèvement de la jubilation devant le retrait du malheur menaçant toujours - c'est cela qui fonde la dignité de cette maison, la dignité du précaire exposé aux violences de l'extériorité. Le delight - ou le plaisir qui naît de l'éloignement du déplaisir, et ce déplaisir encore mais s'éloignant - c'est l'émoi propre à la maison selon Synge. Car les adultes et les enfants qui parlent ensemble dans ce "petit coin de la surface du monde », le font toujours sous la menace de la destruction, et donc dans le ravissement d'échapper présentement à la catastrophe qui toujours se prépare et cette fois-ci se retire. C'est que le dedans de la maison contraste avec son dehors, et s'articule à ce dehors d'une façon telle que la maison entière symbolise la parole humaine, comme ce en quoi les hommes se protègent du péril et de la dernière misère. Dedans, le feu de tourbe délie les langues: le bol de lait ou le verre de whisky - le poteen fabriqué clandestinement - réchauffe les poètes, les penny poets récitant leurs ballades, comme les voyageurs racontant ce qu'ils ont vu dehors. Dehors, c'est ce que Pegeen, dans Le Baladin, nomme the big world : non seulement le continent opposé à l'île

\footnotetext{
${ }^{13}$ Les Îles Aran, , p. 157.
} 
d'Irlande, mais tout l'Extérieur, opposé au domaine propre du paysan irlandais. ${ }^{14}$ Dehors, c'est la nuit : le big world est lourdement proche par la nuit, il pèse sur le toit de chaume comme cette nuit sans égard pour les marcheurs dans les sentiers de boue, la nuit qui est longue en hiver derrière la porte de la cuisine. Et dehors, c'est le vent : or il n'est pas facile de dire ce qu'est le vent d'Irlande. Dans les pièces de Synge, le vent retentit dans les paroles des personnages comme leur crainte de sortir, comme leur angoisse de cet espace infiniment vacant qui cerne la chaumière. La vieille mythologie irlandaise donnait le vent pour symbole non de vanité métaphysique, mais de destruction. Yeats dans son dernier poème, une semaine avant sa mort, La Tour noire (The Black Tower), relance ce symbolisme : "Là-bas dans la tombe les morts très droits se dressent, / Mais les vents montent du rivage : / Ils tremblent quand les vents grondent, / De vieux ossements sur la montagne tremblent. » ${ }^{15}$ Dehors, ce vent et cette nuit condamnent le marcheur à l'épouvante, si durement que, dedans, les récits d'envoûtements par les fées du pays, d'égarements dans la folie, de chute aussi du bétail du haut des falaises, servent à conjurer la peur de perdre l'abri. La maison est sublime de cet énorme espace de dangers autour d'elle. Sublime de la destruction prochaine et encore différée. Sublime de la frugalité du repas où les marcheurs reprennent des forces avant de repartir. Synge fut ce marcheur, allant silencieux par les fossés de la nuit: "Un soir, après de grosses pluies, je me mis en marche en empruntant un sentier escarpé en partie enfoncé dans les bruyères qui traversait les collines me séparant d'un village. » «Une nuit, je devais descendre jusqu'à la ville de Wicklow depuis un village de montagne avant de remonter ensuite dans les collines. » "Un peu plus tard, je leur souhaitais une bonne nuit et repris la route, car j'avais encore deux montagnes à franchir. » ${ }^{16}$ Petite est la maison servant d'abri contre le big world. Délicieuse est la crainte - le delight - que le refuge émeut, de l'espace terrible du vent et de la mer. Sublime est la faiblesse protectrice adossée à la démesure de l'infini : «La chaumière est enveloppée d'un épais brouillard blanc et nous avons l'impression d'être coupés de toute habitation. Tout autour, derrière les collines, le grondement et la rumeur du tonnerre se rapprochent, parfois avec un claquement sauvage et brutal. Le vert des fougères est presque douloureux dans cette lumière étrange. D'énormes moutons passent et repassent sur la ligne du ciel. »17

\footnotetext{
${ }^{14}$ Voir la note de Françoise Morvan à sa traduction du Balladin du monde Occidental, dans : John Millington Synge, Théâtre, Traduit, présenté et annoté par F. Morvan, Actes Sud, Babel, 1996, p. 181.

${ }^{15}$ William Butler Yeats, Derniers poèmes (1936-1939), Édition bilingue Présenté, annoté et traduit par Jean-Yves Masson, Verdier, 1994, pp. 110-113. Nicolas Bouvier, revenu des îles Aran, a témoigné de ce vent à sa façon : "Avec un vent de quatorze Beauforts qui condamne trois mois sur douze les îliens à un mutisme presque total on n'a pas le temps d'enjoliver, on va à l'essentiel. » (Journal d'Aran et d'autres lieux, Payot, 1990, p. 84.)

${ }^{16}$ Pour les trois citations, Dans le Wicklow, L'Ouest du Kerry et le Connemara, p. 35, 44, 52.

${ }^{17}$ Dans le Wicklow, L'Ouest du Kerry et le Connemara, p. 69.
} 
La maison selon Synge apparaît donc comme une parole menacée d'infini, frappée de néant, cette parole pourtant l'abri aussi vivace que précaire contre l'insensé qui l'étonne et qui l'inspire. La phénoménologie de la maison, conduite poétiquement par Synge dans ses récits de marcheur et dans ses drames, s'accomplit ainsi comme l'écoute d'une lamentation - le farouche keen - , cette déploration des morts, plainte extatique dans laquelle s'exalte l'expérience de la terreur du monde et de la désolation sublime. Le verbe to keen semble sans équivalent en français, et Pierre Leyris conserve tel quel le mot keen dans sa traduction. Le keen, donc, se fait entendre dès la première pièce de Synge, lorsque Maurya, la mère qui travaille à la maison, comprend qu'elle a perdu ses six fils, dehors, noyés dans l'éternelle tempête, - et voici le keen :

Ils sont tous partis maintenant, plus rien que la mer puisse me faire... Je n'aurai plus de raison de me lever la nuit pour être à pleurer et prier quand le vent hurle au sud et qu'on entend la houle de l'est, la houle de l'ouest, toutes les deux à cogner l'une dans l'autre, avec le grand vacarme de leurs deux bruits. Plus de raison maintenant de descendre chercher l'eau bénite dans les nuits noires d'après la fête des Morts, plus de raison d'être à s'inquiéter de comment est la mer en entendant les autres femmes commencer à chanter la plainte de mort. $^{18}$

La mélopée de deuil accompagne aussi de sa grande rumeur toute la fin de la dernière pièce, Deirdre of the Sorrows, avant et après la mort de Deirdre. Et quand cette lamentation funèbre retentit en somme dans toute l'Irlande concentrée sur la scène, la vieille servante de Deirdre, Laverkam, invite encore le tyran Conohor, par qui le mal est entré dans le monde, à trouver refuge contre sa propre jalousie, contre sa haine de tous et de lui-même, et contre la nuit qui tombe, dans une chaumière qu'elle $a$, en Ulster : "J'ai une petite maison de terre où tu pourrais prendre repos, Conohor, la rosée tombe dru." (T.315) Le keen et la maison se correspondent. Le keen est la parole sublime de la détresse des pauvres, dans l'ardeur de laquelle ils résistent à l'horreur d'exister, comme la maison est dans son feu de tourbe le lieu où se touchent la destruction du dehors et la chaleur du dedans :

Alors a commencé la sauvage déploration, la plainte de mort. Chaque vieille femme, son tour venu de mener le récitatif, semblait dans l'instant même possédée d'une profonde extase de douleur, se balançant d'avant en arrière,

\footnotetext{
${ }^{18}$ Cavaliers de la mer, dans Théâtre, p. 42.
} 
penchant le front jusqu'à toucher la pierre devant elle, tout en invoquant la morte par un chant de sanglots lancinant, qui renaissait sans cesse. ${ }^{19}$

Le keen est l'expression du lieu sublime. Le keen est enraciné dans la maison entendue comme abri du delight. Le keen, disons, est la maison de l'être. « Le chagrin de la lamentation ne relève pas d'une affliction personnelle [...] mais semble nourri de toute la fureur passionnée qui est tapie quelque part chez tout indigène de l'île. Dans ce cri de douleur, la conscience intime semble se mettre à nu pour un instant et révéler l'état d'âme d'êtres humains qui ressentent leur isolement face à un univers dont les vents et les flots leur font la guerre. Ils se taisent habituellement, mais en présence de la mort tout simulacre d'indifférence ou de patience est oublié, et ils hurlent de désespoir, pitoyablement, devant l'horreur du destin auquel il sont tous condamnés. » ${ }^{20}$

\section{L'Irlande et la langue}

Pression du dehors sur le dedans, pauvreté vécue en dignité, désolation ressaisie en extase ontologique, exposition de la parole précaire à l'extériorité terrible, - la maison selon Synge apparaît d'autre part comme un symbole de l'Irlande et de la condition et du rêve irlandais. Autrement dit, la maison est une synecdoque du pays, et, dans la relation entre maison et Irlande la partie inclut le tout. À la lueur $\mathrm{du}$ feu de tourbe s'éclaire le rapport de Synge - that rooted man - à l'Irlande entendue elle aussi comme la demeure de l'être, comme lieu du delight et de la déploration pour l'existence exposée. Il convient ici de faire valoir la très profonde cohérence et l'économie spirituelle du destin de Synge, en tant que destin irlandais.

D'une part Synge n'est devenu lui-même qu'en vertu d'un retour - retour au pays, à la langue, et à la tradition. D'autre part ce retour n'aura fait qu'accuser l'écart, chez lui inguérissable, entre son désir et son lieu, et n'aura donc été qu'une préparation à la mort. Jeune homme, dès après une licence de langues à Trinity College, il semble atteint de la Wanderlust si fréquente parmi les artistes irlandais, et le voici en Allemagne où il étudie la musique, puis assez vite en France où il suit à la Sorbonne les cours de d'Arbois de Jubainville sur la vieille littérature celtique, étudiant aussi le gaélique et l'ancienne civilisation irlandaise, puis en Italie où il apprend l'Italien, avant de revenir en France pour un apprentissage du Breton. Synge sur le continent voyage entre les langues qu'il assimile aisément - ce qui

\footnotetext{
${ }^{19}$ Texte des Îles Aran traduit par Françoise Morvan et cité par elle en note dans Théâtre, p. 37.

${ }^{20}$ Les Îles Aran,, pp. 47-48.
} 
signale au moins l'ambivalence de sa relation à l'anglais maternel - , comme plus tard il voyagera, revenu dans son pays, à pieds ou à bicyclette (seul ou avec Jack Yeats, le frère de William), entre les mœurs des différents comtés d'Irlande, poursuivant alors son nomadisme en terre natale. Entre l'errance continentale, cependant, et les voyages irlandais, l'énorme différence tient à l'invention d'une langue qui lui est propre et dans laquelle est écrit tout son théâtre. Cette invention, qui constitue sa découverte de lui-même et la signification universelle de son œuvre, n'a été possible qu'à partir de la rencontre des îles Aran.

On sait que c'est Yeats qui incita Synge (leur rencontre eut lieu à Paris dans un hôtel en 1896) à séjourner dans ces îles, " pour y trouver une vie, écrit Yeats, qui n'eût pas été exprimée en littérature $»{ }^{21}$ De fait, c'est à Aran qu'il entreprend de traduire, du gaélique vers l'anglais, l'une des plus vieilles légendes irlandaises, l'histoire de Deirdre, dont il tirera sa dernière pièce; et c'est aussi là-bas qu'il entend les récits dont il fera l'intrigue de toutes ses autres pièces (à l'exception des Noces $d u$ rétameur). Aran, donc, est le lieu de naissance de Synge à son œuvre. Or ce lieu est certes celui de son retour à l'Irlande, mais à une Irlande comme étrangère à ellemême, ou à une Irlande si extrême, et si extrêmement désabritée de son dehors que ce retour apparaît comme un départ. Aran, dans la symbolique des lieux irlandais, et empiriquement dans l'économie du pays, et encore linguistiquement, et quant aux mœurs, c'est à la fois le comble de l'Irlande et tout autre chose, c'est l'Irlande primitive réinventée, mais si primitive que c'est aussi la Grèce antique, et l'Égypte, ou nulle part. Synge a noté plusieurs analogies qui l'ont frappé entre les récits des pécheurs d'Aran et ceux de la mythologie grecque; et Yeats, de son côté, a dit que « les histoires nées de l'art de Synge [étaient] aussi lourdes de sens qu'un cortège peint sur un mur égyptien $» .{ }^{22} \mathrm{La}$ traduction entreprise là-bas atteste qu'il cherchait une langue qui tînt à la fois de l'Irlande empirique, dont son errance et son instruction le séparaient, et de l'Irlande mythique dont Aran était l'évocation.

Si donc Joyce, par exemple, a écrit à partir de l'exil, Synge en revanche a écrit à partir d'un retour-ailleurs (ce dont Joyce du reste crut pouvoir se moquer). Ce retour le conduisant à Aran l'a exposé à des îles intérieures à l'Île natale, des îles en quelque sorte exponentielles, et qui forment une Irlande disons en abîme, où celle-ci ne se connaît qu'en rêve. Yeats s'est souvenu de ce retour au dehors, dans un poème de 1919 :

Et cet homme toujours en quête, John Synge, vient après lui,

\footnotetext{
${ }^{21}$ Cité par Pierre Leyris, « Avant-propos du traducteur », Les Îles Aran, p. 8.

22 «J. M. Synge et l'Irlande de son temps », Le Baladin du monde Occidental, p. 29.
} 
Qui en mourant choisit pour texte le monde vivant

Et n'aurait jamais pu être en paix dans sa tombe

$\mathrm{Si}$, voyageant longtemps, il n'était arrivé

Vers la chute du jour au milieu d'une société solitaire

Dans un lieu très sauvage et rocheux,

Vers la chute du jour au milieu d'une race

De gens simples et passionnés comme son cœur. ${ }^{23}$

Pour comprendre pourquoi Synge s'est rencontré lui-même dans ce lieu très sauvage et rocheux, il faudrait relire la description qu'il en a donnée dans son livre aux "captivantes promesses », comme dit Pierre Leyris, Les Îles Aran. ${ }^{24}$ Ce livre d'abord n'a pu être écrit que par un homme silencieux. Synge, "that rooted man", était aussi, et selon Yeats encore, dans Coole Park, 1929, "that slow man, / That meditative man»; et il a raconté une fois que, «lorsqu'il se trouvait vivre chez les paysans, il essayait de faire en sorte que tout le monde oublie qu'il était là, et il est certain qu'il restait toujours silencieux dans une pièce bondée.» ${ }^{25}$ D'où suit qu'à Aran, il sut aimer «tout ce qui a du mordant, tout ce qui est sel dans la bouche et rudesse rugueuse sous la main, tout ce qui hausse les émotions en s'affrontant, tout ce qui aiguise dans la vie le sens du tragique; et, dans ce livre, [...] il le montre sans penser à rien d'autre qu'à son propre goût. C'est si constant, exposé de manière si simple et si naturelle que cela suggère une correspondance entre une disposition durable de l'âme et cette vie qui partage la dureté du roc et du vent. [...] on sait que son regard est le regard plein d'intérêt affectueux de l'homme simple, non le regard inquisiteur de l'homme d'étude. » ${ }^{26}$ Or les îles, comme la maison, sont sublimes. Leurs traits qui frappent Synge sont les suivants : le danger dans lequel les pécheurs vivent sans arrêt et meurent sur leurs esquifs en peau; la proximité quotidienne de la mort sans autre sépulture que les vagues monumentales; la simplicité confondante des îliens, qui ne savent pas leur courage ni leur incroyable habileté, et qui «ont si peu d'images à leur disposition», qu'ils peuvent croire, voyant des photographies de statues du jardin du Luxembourg, qu'il y a dans les pays du continent « des gens tout nus qui se promènent sans rien sur le dos "; la primitivité de la vie sociale, "peut-être la plus primitive qui reste en Europe" »; la continuité entre le sens du concret et le sens du merveilleux, la fréquentation en particulier naturelle des îliens avec les fées, les sortilèges, les ravissements de l'esprit («Ces gens, dit-il, ne font

\footnotetext{
${ }^{23}$ Yeats, «À la mémoire du commandant Robert Gregory», Les Cygnes sauvages à Coole, Édition bilingue, Traduit par Jean-Yves Masson, Verdier, 1990, pp. 23.

24 « Avant-propos du traducteur », Les Îles Aran, p. 7.

${ }^{25}$ Le poème est reproduit par exemple dans Quarante-cinq poèmes de Yeats, pp. 102-105.

C'est Yeats encore qui en témoigne dans «J. M. Synge et l'Irlande de son temps », Le Baladin du monde Occidental, p. 27.

${ }^{26}$ Le Baladin du monde Occidental, pp. 33-34.
} 
pas de distinction entre le naturel et le surnaturel »; la passion véhémente, en des moments privilégiés, et la faculté poétique, qu'il s'agisse de la mémoire des chansons ou des inflexions de la voix: "[La vieille femme] se balançait sur un tabouret au coin $\mathrm{du}$ feu, derrière un pot d'indigo où elle teignait de la laine, et à plusieurs reprises, lorsque le jeune homme eut fini un poème, elle le reprit en récitant les vers avec une exquise intonation musicale, mettant dans sa voix une passion et un regret qui semblait lui donner tous les accents que nous recherchons dans la poésie la plus profonde. ${ }^{27}$ La lampe avait baissé et de nouveau une terrible tempête sifflait et hurlait sur l'île. C'était comme un rêve d'être assis là, parmi ces hommes et ces femmes, à écouter cette poésie primitive et superbe, emplie des plus anciennes passions du monde. » ${ }^{28}$ Mais encore une fois c'est l'horreur d'exister, et la peine et la misère et la perpétuelle désolation d'un exil impitoyable, d'une vie trop dure, qui donnent à ces îles leur sublimité bouleversante, parce que cette horreur se produit, chaque jour, dans un paysage et une lumière dont Synge note la splendeur inutile, la magnificence déserte, l'écrasante merveille pour rien. Même les oiseaux, à Aran, sont de grands poètes de la dévastation de tout, dans la beauté inconnue du monde, - voici une page très lumineuse :

Beaucoup d'entre les oiseaux déploient devant moi une fatuité de barbares, se livrant à d'étranges évolutions tant que je suis en vue et retournant à leur corniche rocheuse dès que je suis parti. [...] Leur langage est plus facile que le gaélique, et je crois comprendre la plupart de leurs cris, quoique je ne sois pas capable d'y répondre. Il y a une note plaintive qu'ils reprennent au milieu de leur babillage habituel avec un effet extraordinaire et qu'ils se passent de l'un à l'autre le long de la falaise avec une sorte de gémissement inarticulé, comme s'ils se rappelaient pour un instant l'horreur de la brume. / [...] Le passage continuel dans cette île de la désolation d'hier au soir à la splendeur d'aujourd'hui, semble créer une affinité entre les humeurs des insulaires et les alternances de ravissement et de désarroi qui sont fréquentes chez les artistes ainsi que dans certaines formes d'aliénation. Pourtant c'est seulement dans l'intonation de quelques phrases ou dans une vieille bribe de mélodie que je saisis le véritable esprit de l'île, car en général les hommes s'asseyent de compagnie pour parler, avec des redites sans fin, de la marée, du poisson et du prix de la soude au Connemara. ${ }^{29}$

\footnotetext{
${ }^{27}$ Les Îles Aran, p. 114.

L'Ouest du Kerry et le Connemara, p. 96.

Les Illes Aran, p. 23.

Les Îles Aran, p. 113.

${ }^{28}$ Les Illes Aran, pp. 91-92.

${ }^{29}$ Les Îles Aran, p. 46.
} 
Comment comprendre, maintenant, que ce fût parmi ces îliens au « désenchantement préhistorique», que Synge pût rencontrer sa propre langue, et avec elle la juste distance entre son désir et l'Irlande? ${ }^{30}$ Il faut ici interroger l'isolement douloureux, et relativement assumé, dans lequel il vécut là-bas, ne connaissant avec ses hôtes, qui le nommaient volontiers "noble étranger", que quelques moments de communion - dans les périls de la mer, dans des marches contre le vent ou dans l'étonnement des paroles du soir -, mais portant d'ordinaire le poids de ses différences et de l'incompréhension réciproque. Jamais Synge n'a pu confondre Aran avec une Arcadie, et jamais n'a-t-il seulement pu croire qu'y vivre lui serait possible, bien qu'il eût avec certitude la conscience que ce confin du monde, hors des civilisations, hors des signes qui recouvrent la vie, hors des médiations culturelles, était d'emblée le lieu de son accès à lui-même et de son acceptation de soi, seulement frappé pour lui d'une impossibilité personnelle. Un poème de la fin de sa vie ressaisit le souvenir de cette identité trouvée dans cette impossibilité tragique :

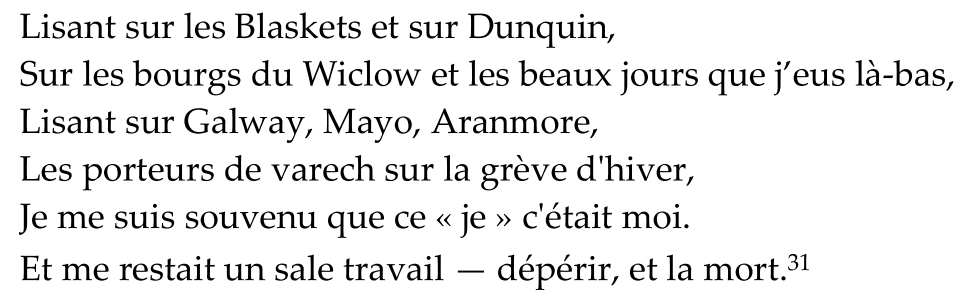

L'écart de Synge par rapport aux îliens se laisse deviner dans cette reconnaissance finale, et ce devoir de solitude. À Aran, il bute sur l'incommunication : "Je suis davantage, dit-il, en communion de sentiments avec eux qu'ils ne peuvent l'être avec moi, et tandis que j'erre parmi eux, tantôt je leur plais, tantôt ils se moquent de moi, mais ils ne comprennent jamais ce que je fais. » ${ }^{32} \mathrm{Ce}$ qu'il fait, c'est le travail invisible de sa conscience de soi et de l'invention de sa parole propre. Ce travail est à ce point séparé, distant des intéressements ordinaires de l'urgence quotidienne, que Synge l'aura perçu comme un «sale travail » (a filthy job), celui d'accentuer encore, dans l'expérience du monde, l'affleurement du réel sous les mots, celui de désencombrer encore l'esprit de ses acquisitions, de ses savoirs spéciaux et de ses idées abstraites : travail de dévastation des codes (to waste) en totale continuité avec la terre nue des îles d'Aran, mais travail d'intériorisation de la finitude personnelle ((to waste

\footnotetext{
${ }^{30}$ Les Îles Aran, , p. 93.

${ }^{31}$ Je cite ici ma traduction: John Millington Synge, Douze poèmes, Choix, traduction et présentation par Jérôme Thélot, Montpellier, Éditions Grèges, p. 31.

${ }^{32}$ Les Îles Aran, p. 93.
} 
and die) qui isole de ses hôtes le «noble étranger ». Synge a distrait les îliens par des trouvailles plus ou moins drolatiques, dissimulant son travail invisible : il leur a montré des photographies, qu'il prenait d'ici et d'ailleurs; il a joué, pour qu'ils dansent, à son violon; il a captivé leur attention par des tours de prestidigitation; il leur a raconté ses voyages dans le big world. En échange : leur affection étonnée ou moqueuse, leur reproche de le voir toujours célibataire, et l'enseignement de leur dialecte. "Sous la sympathie que nous éprouvons, dit-il d'une jeune fille, il n'y en a pas moins un abîme entre nous. » ${ }^{33}$ Du reste il a quitté Aran - ce que les îliens ne font pas, sinon pour New-York - et il est revenu pour repartir, et revenu encore : quatre séjours auront aggravé l'une par l'autre la désertion, là-bas, des médiations européennes, et la retrouvaille, à Dublin ou à Paris, avec la littérature d'Irlande.

"Je n'ai jamais entendu parler plus simple et plus attrayant que celui de ces îliens.» ${ }^{34}$ Par l'isolement moral, par l'écart des cultures, par l'apprentissage du dialecte local, par la traduction en anglais du mythe irlandais, et par l'adhésion au parler simple des insulaires les plus séparés d'Europe, Synge est entré dans sa vraie manière et a inventé sa langue, - sa langue aussi résolument extrême que paysanne, aussi enracinée que singulière, susceptible d'être dite par un comédien des scènes européennes quoique hyperboliquement irlandaise. La langue de Synge est fidèle à l'écart par rapport à tout lieu, dans lequel son inventeur a vécu. Elle est langue de l'écart, sans commune mesure, et langue de la déploration interne à l'écart : langue pour la simplicité du sublime, pour la maison trempée de détresse, et pour le keen. Joyce a d'abord fait remarquer que jamais en Irlande il n'avait entendu personne s'exprimer comme un paysan des pièces de Synge, et il donnait cette remarque pour une objection, comme si le réalisme apparent de ces pièces avait dû commander un décalque populiste des formes linguistiques, - mais c'était ne s'irriter que de l'écart interne à l'invention poétique. ${ }^{35}$ Cette langue, davantage éloignée de l'anglais académique que du parler paysan $\mathrm{du}$ western world, est un anglo-irlandais, un kiltartan: un anglais, donc, mais parlé par des sujets qui pensent en gaélique, un anglais du coup dévasté d'autres structures travaillant en lui, comme le souvenir d'un autre pays occupe la mémoire en terre étrangère, - un anglais, faut-il dire, disloqué, où se fait entendre l'habitation ambivalente et déchirée d'une conscience que se partagent

\footnotetext{
${ }^{33}$ Les Îles Aran, p. 94.

${ }^{34}$ Les Illes Aran, p. 132.

${ }^{35}$ Joyce révisa du reste son jugement et collabora à une traduction de Riders to the Sea en italien, et l'on connaît dans Ulysse la réponse de Buck Mulligan à qui l'on a demandé sa théorie sur Shakespeare : «- Shakespeare? dit-il. Je crois que j'ai entendu ce nom-là. / Un fugace sourire ensoleilla sa face épaisse. / — Eh parbleu, dit-il triomphant, ça me revient. Le type qui a écrit à la manière de Synge. » (Joyce, Ulysse, Traduction d'Auguste Morel revue par Valéry Larbaud, Stuart Gilbert et l'auteur, Gallimard, 1957, Folio, t. I, 1972, p. 286.)
} 
son rêve gaélique et son histoire anglophone, sa culture européenne et son extase insulaire, son nomadisme et son enracinement. Cet anglo-irlandais, comme dit Françoise Morvan, «n'est pas utilisé [...] comme patois, comme curiosité exotique ou parler barbare mais comme langue double, langue du partage intérieur, [et] de l'impossible présence au monde. ${ }^{36}$ Création poétique au confluent de deux langues, par quoi Synge devient lui-même, non plus en se disant (comme dans ses poèmes de jeunesse), mais en laissant retentir les tensions de la vie paysanne, les contradictions du dedans et du dehors, et où la singularité de son écart personnel trouve à s'universaliser en une parole bâtarde. Encore une fois Yeats apprécie comme il convient cette invention : «Peut-être aucun paysan irlandais, dit-il, n'a t-il eu exactement ce rythme-là dans la voix, mais certainement si M. Synge était né paysan, c'est ainsi qu'il aurait parlé. » ${ }^{37}$ (FS 25)

\section{Pour une poétique de la maison.}

L'intrication de l'anglais et du gaélique dans la langue disloquée coïncide avec l'intrication de l'intérieur et de l'extérieur dans la maison sublime. La maison et la langue se correspondent et se métaphorisent réciproquement dans cet écart intime à chacune, où elles trouvent leur vérité tragique. La langue comme la maison, la maison comme la langue, est une intimité envahie, et soufflée, par son dehors qui la tourmente et la nourrit, un feu qui flambe au foyer d'ici par la faveur dangereuse de l'air immense là-bas. Dans cette clef, peut se lire tout le théâtre de Synge, comme ce feu et comme un seul poème. Voici pour finir quelques brefs aperçus.

Dans la première pièce, Riders to the Sea, la mort vient aux hommes sortis de la maison pour pécher en mer, et la mort entre dans la maison par le bruit de la déploration des femmes; la dernière phrase du drame, dite par la vieille mère en deuil de tous ses fils, atteste la compassion dont l'œuvre entière est animée : "Aucun homme au monde n'est fait pour vivre toujours, et nous devons être contents. » 38 Dans In the Shadow of the Glen, écrite en même temps, une jeune femme recrue de solitude au fond de la vallée et ravagée d'attendre que passe par sa maison quelqu'un qui pourrait la désirer, accueille complaisamment un étranger qu'elle attire jusque dans sa chambre, de sorte que son mari la chasse et la voici au moment de sortir, contrainte au dehors, exposée pauvre à l'errance des vagabonds, - mais l'étranger l'encourage : «Venez avec moi, dame de la maison, c'est pas que

\footnotetext{
${ }^{36}$ Fr. Morvan, «Introduction », dans J. M. Synge, La Fontaine aux saints, Traduction, présentation et notes de Fr. Morvan, Éditions Folle Avoine, 1995, p. 15.

${ }^{37}$ Yeats, «Préface à la première édition de La Fontaine aux saints, p. 25.

${ }^{38}$ Théâtre, p. 44.
} 
mon jabotis que vous entendrez de ce temps qu'on ira - c'est le cri des hérons qui volent sur les lacs noirs, et le coq de bruyère, et les hiboux avec, et les alouettes et les grives draines les jour qu'il fera chaud - [...] c'est rien que des chansons fines que vous entendrez d'eux au lever du soleil [...]. »39 Alors les deux pièces suivantes, The Well of the Saints et The Tinker's Wedding, sont celles en effet du dehors, présentant, l'une, les mendiants aveugles («Une région solitaire dans les montagnes à l'est de l'Irlande »), l'autre les rétameurs («Le bas-côté d'une route de village à la tombée de la nuit»), les plus pauvres parmi les pauvres et sans autre refuge que leur parole, mais celle-ci alors tout à fait géniale, grevée des choses du monde, concrète à craquer, saturée de toute l'énergie du ciel et de la boue des chemins, - ou, comme disait Yeats, enracinée à en perdre les mots. ${ }^{40}$ Ces deux piècesci témoignent du travail d'appauvrissement, de dépouillement des savoirs, entrepris par Synge, et métaphorisé ici par l'acceptation du dehors, dans l'horreur et la joie d'une vie sans maison. Les deux dernières pièces, The playboy of the western world, et Deirdre of the Sorrows, sont de très grandes œuvres d'une puissance formidable. Dans le Playboy, la même situation que dans In the Shadow of the Glen se reproduit, d'un vagabond entré dans la maison et soulevant de sa parole l'énorme beauté du monde. Dans Deirdre of the Sorrows, au contraire, il revient à la plus sauvage et la plus belle des jeunes filles, promise malgré elle au roi, de devoir quitter sa maison et de fuir même l'Irlande pour vivre dehors avec son amant sous les arbres d'Écosse, jusqu'au jour du retour qui sera jour de la mort. Cette dernière pièce fut pour Synge sa préparation à sa mort, tout retour à jamais proscrit; on $\mathrm{y}$ comprend que l'Irlande elle-même, la « déesse » dont il était jadis tombé amoureux, lui fut interdite, comme lui fut interdit le foyer simple qu'il a toujours souhaité. Yeats dit la vérité sur son ami aussi partout étranger qu'évidemment situé : "l'on dirait [...] un homme né ailleurs, très loin dans l'immensité de l'espace et du temps. ${ }^{41}$

\footnotetext{
${ }^{39}$ Théâtre, p. 64.

${ }^{40}$ Théâtre., p. 70.

Théâtre p. 132.

${ }^{41}$ « J. M. Synge et l'Irlande de son temps », Le Balladin du monde Occidental, p. 35.
} 